\title{
Recognition and recall of invisible objects
}

\author{
IAN BEGG and MARYANN AZZARELLO \\ McMaster University, Hamilton, Ontario, Canada
}

\begin{abstract}
Three experiments examined recognition and recall of items that people imagined as being invisible. When one item hid another, the hidden item suffered in recognition, but so did the one that remained visible; the demand to imagine items invisibly made both items less recognizable than in control conditions. Cued recall did not depend on whether the visible item or the invisible item was the cue, but it did depend on whether the initial task required relational processing of the items. When both items were invisible in a dark imaginary scene, there was a general loss in all measures of memory, including memory for the scene. None of the results requires recourse to the "out of sight, out of mind" principle that memory depends on the visual characteristics of images. The results attest, instead, to the importance of the informational characteristics of memorial records.
\end{abstract}

Our concern in this study was with people's memory for items that they have imagined in different ways. There is much evidence that images have perceptual properties that influence cognitive behavior in tasks such as mental rotation (Shepard \& Metzler, 1971), symbolic comparison (Paivio, 1975), and sentence verification (Glass, Millen, Beck, \& Eddy, 1985). In addition, people answer questions about imagined details faster if objects are imagined as large rather than small (Kosslyn \& Pomerantz, 1977). Furthermore, tasks that depend on images use the same resources as explicitly perceptual tasks, inasmuch as there is modality-specific interference between the two kinds of tasks (Brooks, 1968; Paivio, 1975; Segal \& Fusella, 1970). Images certainly have visual properties that influence cognitive behavior. Our thesis is that it is not because images have visual properties that imagery is associated with memory.

Imaginal encoding has much in common with other encoding processes that require permanent knowledge to satisfy explicit demands. Imagining a bird requires tacit knowledge about feathers, beaks, and eyes, harmoniously blended into a coherent form. Our thesis is that the process of using and blending knowledge is more important for memory than the fact that the image has a quasi-perceptual reality.

In simple form, the question is whether "out of sight, out of mind" describes memory. Luria's (1968) mnemonist failed to recall egg, and explained that he could not "see" the egg during retrieval because he had origi-

This research was supported by Grant A8122 from the Natural Sciences and Engineering Research Council of Canada to Ian Begg. The data were collected in two independent studies courses by Maryann Azzarello, supervised by Ian Begg. The paper was written while Ian Begg was on sabbatical leave at Erindale College of the University of Toronto. We thank Fergus Craik, Meredyth Daneman, and the members of their research groups for their discussions of the experiments. We also thank Ann Anas, Marcia Barnes, Paula Hertel, Marc Marschark, and Andrea Snider for their helpful comments on the paper or the issues. Reprints can be obtained from Ian Begg, Department of Psychology, McMaster University, Hamilton, Ontario L8S 4K1, Canada. nally imagined it against a white background. However, a satisfactory test needs a contrast between imaginal processes that use the same cognitive machinery, but whose imagined products differ in their visual appearance.

Begg (1983) used insubstantial or substantial adjectives with nouns, as in invisible butterfly and colorful butterfly, to vary imaginal appearance. The results did not suggest "out of sight, out of mind"; insubstantially modified nouns were the better recognized, insubstantial adjectives were the better cues for recall of their nouns, and both measures were better if people were instructed to imagine the phrases than if they were not. The results may reflect greater difficulty in processing the insubstantial phrases (e.g., Jacoby, Craik, \& Begg, 1979), but it is hard to explain them on the basis of visual properties of images.

Another way to influence visual appearance is to have people imagine items that are concealed. Neisser and Kerr (1973; Kerr \& Neisser, 1983) examined recall of items such as clock, cued by sentence contexts. The sentences described scenes in which the context and target interacted (clock was on the top of a snowbank), or they were separate (clock was on a nearby mountain), or the context concealed the target (clock was buried under the snowbank). Because concealed items were recalled nearly as well as interactive ones, Neisser and Kerr rejected the idea that imagery is "mental seeing." They argued for "spatial knowing," by which the spatial layout required to perform the concealment task is useful for recall. The argument gains additional support because the results are about the same for blind subjects as for sighted subjects (Kerr, 1983; Zimler \& Keenan, 1983). However, concealed items are not recalled better than separate ones if there are subtle changes in the procedure (Keenan \& Moore, 1979; see Keenan, 1983, and Kerr \& Neisser, 1983, for further discussions).

The present experiments examined memory after people imagined scenes that included invisible objects. The measures included recognition of invisible and visible objects, and recall of visible and invisible objects cued by 
visible and invisible objects. The instructions included several ways of making objects invisible and several control procedures. We found that the visual properties of the images had no unique consequences for memory.

\section{Organization-Redintegration Hypothesis}

We propose that imagery instructions influence encoding in the same lawful manner, whether or not the instructions specifically demand invisibility. Cued recall depends on whether the items are encoded in relation with each other, and recognition depends on whether each item is encoded in a distinctive way that is accessible during the test. Neither measure depends on whether the item is invisible, but rather on how the item was encoded in the course of making it invisible.

The organization-redintegration hypothesis (Begg, 1982) views encoding as the active process of organizing items. If the task is to imagine the members of a pair interacting with each other, each pair is encoded as a single memory unit. If the task is to imagine each member as a separate image, each pair is encoded as two independent memory units. If one member of an interactive pair retrieves its unit, redintegration of the unit makes the other item available, but if one member of a separately imagined pair retrieves its unit, redintegration provides no information about the other item. Therefore, cued recall is better after interactive imagery than after separate imagery (Begg, 1973, 1978a; Begg \& Anderson, 1976; Begg \& Young, 1977; Bower, 1970; Dempster \& Rohwer, 1974).

Interactive imagery is a good way to link two otherwise unrelated items, but nonimaginal relational processing also aids recall. If subjects relate pairs by making verbal associates or repeating jointly (Begg, 1983) or by listing ways the items are similar (Begg, 1978b), cued recall is better than if each item is processed separately. Thus relational processing at study binds items into memory units that allow recall of additional items once the unit has been retrieved.

Our hypothesis assumes that relational processing is causally independent of how distinct each item is in the unit. For instance, imagining bird interacting with desk does not make either item less distinct than imagining each alone. In many experiments, recognition has been equally good after interactive and separate imagery (Begg, 1978a, 1982; Begg \& Sikich, 1984; Bower, 1970; Dempster \& Rohwer, 1974). There also have been many experiments in which separately imagined items have been recognized better than interactively imagined items (Baker \& Santa, 1977a, 1977b; Begg, 1979, 1983; Begg, Maxwell, Mitterer, \& Harris, 1986; McGee, 1980). Although relational encoding does not cause items to be encoded less distinctively, the demand to relate items may bias encoding so that the items are specific to their contexts. For example, the demand to imagine mother interacting with railroad may cause a different encoding of mother from when mother appears alone as a cue.

In summary, an item is recognized if it retrieves a unit that has enough item-specific information to identify the item. Cued recall succeeds if the unit retrieved by the cue links the cue and the target. The fact that the items share a unit does not cause either one to be poorly identified, but relating them increases the chance that each item will be encoded differently from when it is alone on a test. Similarly, the fact that items are invisible has no causal consequence for item-specific identification or interitem relations. The demand to imagine invisible objects, however, influences encoding of items and relations. Recall will be better if the task requires relational rather than separate processing, but recognition will favor tasks that require independent processing of the items.

\section{EXPERIMENT 1}

All subjects in Experiment 1 studied pairs of nouns whose members appeared sequentially. The subjects imagined the first item, and then imagined the second item in one of several ways. There were six "invisible" conditions and four control conditions.

We chose control conditions to obtain a reasonable range of recognition and recall to compare with the invisible conditions. Two control conditions were interactive imagery and separate imagery; cued recall has been shown to favor interactive study, but recognition favors separate study, especially for the second items of the pairs (Begg, 1983). Another control condition was successive imagery; if two items are imagined separately from each other in the same scene, recall is between interactive and separate imagery (Begg \& Sikich, 1984). In the other control condition, subjects were instructed to make each image so large that it filled its scene; the task biases independent processing of the two items, so that recall and recognition will be about the same as after separate imagery.

In the six invisible conditions, the subjects were to imagine scenes in which only one of the two items was visible. There were three ways of doing this, each with either the first or the second item visible. The three instructions to make one item invisible were insertion, relative size, and superimposition. Insertion means that one image is put into another; insertion requires joint consideration of the two members, especially if the second item is inserted into the first. Relative size means that one image is so large that the other cannot be seen; making either image large can be done independently for the two items. Superimposition means that one image is in front so that the other one cannot be seen; placing the second image behind the first requires more joint processing than relative size, but placing the second in front can be done with little reference to the first. We chose these three methods on the basis of our intuitions that they demand different degrees of joint processing.

First and second items were expected to behave differently. Because the first items in each condition were initially imagined independently, we expected subjects to recognize them equally well whether they were visible or invisible in the final image. In contrast, second items 
were imagined independently in only some conditions. We expected that second items would be recognized more poorly than first items only if the task biased encoding them differently from when they were alone. If the second item was hidden by the first, it was imagined with reference to the first, but if the second item was visible, it could have been imagined independently of the first one. Thus visible and invisible first items were not expected to differ in recognition, but visible second items were expected to be recognized better than invisible ones, and recall was expected to favor having the second items hidden by the first ones.

According to the "out of sight, out of mind" hypothesis, visible items will be recognized better than invisible ones, and recall will be poor regardless of which item is invisible.

\section{Method}

Subjects. One hundred students of introductory psychology at McMaster University served as a requirement of the course. Ten subjects were assigned at random to each of 10 conditions.

Materials. One hundred sixty-eight nouns with imagery ratings (I) $>6$ and frequencies (F) $>9$ were chosen from Paivio, Yuille, and Madigan (1968) to make a study list and a test. The nouns were sorted into seven sets of 24 items. Each set had a mean $I=6.47$, and the same number of items with frequencies of $\mathrm{AA}, \mathrm{A}$, and 10 to 49 . One set made 12 pairs of untested fillers; 6 were at each end of the list. Two sets provided 48 distractors for the test. The other four sets were assigned at random to be the 48 first and 48 second items; the cues on the final test were the first members of 24 pairs and the second members of the other 24 pairs. Thus the list had 60 pairs, of which the middle 48 were tested. The list was recorded on videotape, with $7 \mathrm{sec}$ between pairs. Each pair was introduced by a row of asterisks; then the first item appeared on the left side of the screen for about $3 \mathrm{sec}$, and then the second item appeared alone on the right side for about $3 \mathrm{sec}$.

The test had eight blocks of 12 items. Each block had 6 distractors, 3 first items, and 3 second items. All 48 pairs were tested once. Each word had the letters $\mathrm{Y}$ and $\mathrm{N}$ to its left and a blank space to its right.

Procedure. ${ }^{P}$ The subjects were encouraged to try to follow instructions. All testing immediately followed study; on the test, the subjects were to circle $Y$ for old items and to recall their partners if they could, and to circle $\mathbf{N}$ for new items. The test was self-paced and took less than $5 \mathrm{~min}$.

There were different instructions for the four control and the six invisible conditions. The control instructions are described first.

1. Interactive imagery. These subjects imagined the first word of each pair and projected it to an imaginary frame above the monitor; they then imagined the second item interacting with it.

2. Successive imagery. These subjects imagined each first item and projected it to an imaginary frame above the monitor. They repeated the process for the second item, projecting the image above the monitor.

3. Large images. These subjects imagined each first item and projected it to an imaginary frame above the monitor. They did the same for the second item, with each image so large that it filled the entire frame.

4. Separate imagery. These subjects formed an image for each word, projecting the image of the first item to an imaginary frame to the left of the monitor, and the image of the second item to the right side.

Instructions for the other six conditions asked subjects to make one item invisible. "Ab" means the first item was to be visible and the second invisible; " $\mathrm{aB}$ " means the second item was to be visible and the first invisible.

5. Ab insertion. These subjects imagined the first item, then imagined inserting the image of the second one into the first so that only the first remained visible.

6. $a B$ insertion. These subjects imagined the first item, then imagined inserting it into the second so that only the second remained visible.

7. Ab superimposition. These subjects imagined the first item, then imagined that the second item was behind it so that only the first remained visible.

8. $a B$ superimposition. These subjects imagined the first item, then imagined the second one in front of it so that only the second was visible.

9. Ab relative size. These subjects imagined that the first item was so large that it filled the frame, and then retained that image while imagining that it completely hid the image of the second item.

10. $a B$ relative size. These subjects imagined each first item, then imagined the second item filling the entire frame so that the first image was no longer visible.

\section{Results and Discussion}

The purpose of the analyses was to determine the costs of invisibility on recall and recognition. Means are proportions, and $M S$ Se values are squared proportions. The $\alpha$ level was .05 for all inferences; simple effects were evaluated by critical differences from post hoc $t$ tests. Mean recognition of the cues and recall of their partners are in Table 1.

Recognition hits are in the first two columns; false alarms did not differ reliably over the conditions $(M=.11, M S e=0.010)$. Analysis of hits revealed a main effect over the 10 conditions $[F(9,90)=2.68, M S \mathrm{Se}$ $=0.0063]$, a main effect of item $[F(1,90)=65.7, M S e$ $=0.0079]$, and an interaction between the two variables

Table 1

Recognition and Recall for Experiment 1

\begin{tabular}{|c|c|c|c|c|c|c|}
\hline & \multicolumn{3}{|c|}{ Recognition } & \multicolumn{3}{|c|}{ Cued Recall } \\
\hline & A? & B? & A-B & A?B & $\mathrm{B} ? \mathrm{~A}$ & $M$ \\
\hline \multicolumn{7}{|c|}{ Control Conditions } \\
\hline Interactive Imagery & .90 & .73 & .17 & .37 & .41 & .39 \\
\hline Successive Imagery & .92 & .79 & .13 & .14 & .18 & .16 \\
\hline Large Images & .90 & .87 & .03 & .08 & .10 & .09 \\
\hline Separate Imagery & .92 & .84 & .08 & .05 & .08 & .07 \\
\hline$M$ of Control & .91 & .81 & .10 & .16 & .19 & .18 \\
\hline
\end{tabular}

Invisible Conditions

\begin{tabular}{|c|c|c|c|c|c|c|}
\hline $\mathbf{A b}$ & & & & $\mathrm{A} ? \mathrm{~b}$ & b?A & $M$ \\
\hline Insertion & .80 & $.65^{*}$ & 15 & $.27^{*}$ & .35 & .31 \\
\hline Superimposition & .78 & $.70^{*}$ & .08 & $.17^{*}$ & 19 & .18 \\
\hline Relative Size & .85 & $.64 *$ & .21 & $.13^{*}$ & .13 & .13 \\
\hline$M$ of $\mathrm{Ab}$ & .81 & $.66^{*}$ & .15 & $.19^{*}$ & .22 & .21 \\
\hline $\mathrm{aB}$ & & & & a?B & B?a & $M$ \\
\hline Insertion & $.80^{*}$ & .77 & .03 & .20 & $.21 *$ & .21 \\
\hline Superimposition & $.80^{*}$ & .70 & .10 & .11 & $11^{*}$ & .11 \\
\hline Relative Size & $.83 *$ & .78 & .05 & .13 & $.17^{*}$ & .15 \\
\hline$M$ of $\mathrm{aB}$ & $.81^{*}$ & .75 & .06 & .15 & $.16^{*}$ & .16 \\
\hline$M$ of Invisible & .81 & .71 & .10 & .17 & .19 & .18 \\
\hline
\end{tabular}

${ }^{*}$ Invisible item. 
$[F(9,90)=2.86]$; any two means that differ by .08 are reliably different.

All first items were imagined before the appearance of their partners. The 10 means for recognition collapse into two; the four control conditions did not differ among themselves (.91), but they exceeded the six invisible conditions, which did not differ among themselves (.81). In the invisible conditions, recognition of the visible first items (.81) was the same as that of the invisible first items $(.81)$. Thus the demand to imagine items invisibly reduces recognition, but the cost is equally great for invisible and visible items, and for each method of making the items invisible.

Second items were also recognized better in the control than in the invisible conditions $(.81>.71)$, but the means are misleading because there were differences across the conditions. For the controls, second items were recognized better if they were separate than if they were interactive $(.84>.73)$, and if they were large than if they were successive $(.87>.79)$. In the invisible conditions, second items were recognized better if they were visible than if they were invisible $(.75>.66)$; this difference did not occur for superimposition. The poor recognition of the invisible second items is not because of their invisibility; they were worse than the control values, but so were their visible partners, and the difference was about the same.

The third column is the difference between recognition of first and recognition of second items; the average decline was . 10 for both the control conditions and the invisible conditions, but the averages ignore different declines within each set of conditions. The second item declined .15 if it was invisible (the Ab conditions), but it also declined .15 if it was interactive or successive. It declined only .06 if the second item was visible (the aB conditions), but it also declined .06 if it was separate or large. In other words, processing the second item with reference to the first increases the chance that the second item will be encoded differently from when it is processed on its own. Processing the second item independently of the first item means that the item is encoded more as it will be on the test.

In sum, recognition shows a general cost from imagining items invisibly, but the cost is to both members of pairs, whether they are visible or invisible in the image. Recognition of second members of pairs declines, but the loss is small if they are separate, large, or visible in the image. The loss is larger if they are interactive, successive, or invisible.

Cued recall results also appear in Table 1. The Mean column in Table 1 is averaged over the cues; recall was slightly better if cued by second than by first items $(.19>.16)[F(1,90)=7.01, M S e=0.0054]$, but cue did not interact with condition. The 10 conditions differed $[F(9,90)=6.71, M S e=0.0075]$; the critical difference for simple effects is .06 .

Recall was unlike recognition in several ways. There was a general cost of imagining invisibly for item-specific recognition but not for relational recall; mean recall was
.18 both in the control and in the invisible conditions, but there were large differences within each set of conditions. In the control conditions, recall of interactive images exceeded that of separate images, recall of successively imagined pairs fell between that of interactive and that of separate imagery, and large images were recalled as poorly as separate images. There are thus three values to compare with the other conditions- $.39, .16$, and .07reflecting declining degrees of relation.

Recall in the invisible conditions differed. Insertion had the best recall, especially if the first item enclosed the second (.31), rather than if the second enclosed the first (.21). Superimposition favored placing the second item behind the first rather than placing the second item in front of the first $(.18>.11)$, but relative size was insensitive to which item was visible (.13 vs. .15). Only recall of pairs using insertion exceeded recall after successive imagery; all the invisible conditions exceeded separate imagery, but the case with the second item in front of the first did not do so reliably (.11 vs. .07).

In sum, cued recall varied with how items were imagined. Recall favored having the first item visible, and it favored insertion as the way to hide one item with another. It did not matter whether the invisible item was the cue for a visible partner (.19 for $a$ ? $B$ and $b$ ?A) or if the visible item sought an invisible partner (.18 for $\mathrm{A}$ ?b and $B$ ?a).

\section{Conclusions}

Experiment 1 compared six ways of imagining invisibly and four control procedures. The demand to make one image invisible hurts recognition of both items. Recognition of invisible second items is worse than recognition of their visible partners, but the shortfall is no greater than if the second item is imagined in interaction with the first. Recognition of visible second items is not much worse than recognition of their invisible partners, and recognition of large or separate second items is not much worse than that of their partners. Thus invisibly imagining items reduces the quality of item-specific information. Independent processing of the second item-imagining it as large, or separate, or visible-yields better recognition than processing the second item in relation to the firstimagining it interacting with or hidden by the first.

Recall, unlike recognition, had no general cost of the invisible conditions over the control conditions. Recall varied with how the items were made invisible, approaching interactive imagery at the high end and separate imagery at the low end. The second item can be made visible with relatively little reference to the first item, and recall was lower than if the first item stayed visible, a task that demands joint consideration of both items. Insertion more specifically entails relational processing than does making one item large or close, and it yields better recall. Recall reflects the extent to which the task requires relational processing.

The results do not argue that the visual properties of images underlie memory performance. We propose that the functional properties of imaginal encoding affect 
memory. Visible and invisible partners do not vary in recognition because of their visual role in the depiction; they differ because the process of forming the picture influences the encoding of knowledge specific to the items. The visual roles of the items do not influence recall; what matters is whether the initial process entails joint encoding of the items.

\section{EXPERIMENT 2}

Experiment 2 kept four conditions from Experiment 1 . Three were controls, namely interactive imagery, separate imagery, and successive imagery; the large imagery condition was almost identical in results to the separate imagery condition, so it was dropped. The invisible condition that was retained was the one in which the first item was so large that the second item could not be seen; because this was the condition with the poorest recognition of the invisible second item, one could argue that this is the condition in which invisibility was most effective in reducing recognition.

For Experiment 2, we chose small items that are easy to hide, such as nail, ticket, and lemon, and large ones that can easily hide them, such as door, ocean, and church. These items were paired such that the large one was imagined first and the small second; it is easy to imagine a door large enough to hide a lemon.

Another addition to Experiment 2 was a direct contrast between interactive and separate imagery for visible or invisible pairs. The subjects were instructed to perform each task with the sun shining so that all objects were clearly visible, or at night so that the objects were present but could not be seen. If the visual qualities of images influence memory, objects imagined in the dark should suffer in recognition, and the difference in cued recall between interactive and separate imagery should be removed by darkness. We expected that interaction in the dark would exceed separation in the dark in cued recall, because of the relational processing done while making them invisible.

\section{Method}

Subjects. One hundred sixty students from the same pool used in Experiment 1 served as subjects, with 20 in each of eight conditions.

Materials and Procedure. All subjects studied 60 pairs of nouns. The first 4 and last 4 were untested fillers. The list was recorded on videotape with $7.5 \mathrm{sec}$ for each pair. Each cycle had a row of asterisks ( $1 \mathrm{sec})$, a blank interval $(.5 \mathrm{sec})$, the first item on the left side $(2.5 \mathrm{sec})$, a blank interval $(.5 \mathrm{sec})$, the second item on the right side $(2.5 \mathrm{sec})$, and another blank interval $(.5 \mathrm{sec})$.

The subjects studied the list in one of eight ways. Two interactive groups imagined the items from each pair interacting in a frame above the television monitor. Two successive groups imagined the first item in the frame, then imagined the second item in the same frame but not interacting with the first. Two separate groups imagined the first item in a frame to the left of the monitor, then the second in a frame to the right. The two relative size groups imagined the first item in a frame above the monitor, with the image entirely filling the frame; the second item was then imagined behind the first so that it could not be seen.

The two groups using each type of imagery differed in the context they were to imagine. One group was to imagine the objects in scenes in which bright sunlight made the objects clearly visible, and the other group was to imagine the objects in scenes in which the darkness of night made the objects invisible. We later refer to these contexts as light and dark, respectively.

The items were 146 nouns, with $I \geq 6$, taken from Paivio et al. (1968). We chose 39 large items (e.g., church, ocean, door) and 39 small ones (e.g., nail, lemon, ticket). Thirteen of each were selected at random to be distractors in recognition, and the remainder were paired at random to make 26 special pairs with a large first item and a small second item; of these, 13 were tested with the large items as cues, and the other 13 were tested with the small items as cues.

The remaining 68 nouns were paired at random to make 8 filler pairs and 26 random pairs. The list began with 4 filler pairs, then the 26 special pairs, then the 26 random pairs, then 4 fillers. The tests followed immediately. The subjects had $5 \mathrm{~min}$ to recall the random pairs; 13 of the first and 13 of the second items were cues, with a blank space next to each one for recalling its partner. The reason for the random pairs was to increase the length of the list and of the retention interval before testing the special pairs; the random pairs will not be discussed in the results. The second test had 26 old items, the large items from 13 pairs and the small items from the other 13 pairs, and 26 distractors. The subjects had 5 min to circle $\mathrm{Y}$ or $\mathrm{N}$ (old or new) for each item and recall the partners of the old cues.

\section{Results and Discussion}

As in Experiment 1, the aim was to determine the costs of invisibility. Mean recognition and cued recall are in Table 2 .

Recognition hits are in the left two columns; false alarms did not differ reliably $(M=.15, M S e=0.018)$. The means in Table 2 are averaged over light and dark contexts; recognition was equal in the two (.75 vs. .73), and no interaction with this variable was reliable. Thus, contextual darkness did not reduce item-specific encod-

Table 2

Recognition and Recall in Experiment 2 as a Function of Study Context and Type of Imagery

\begin{tabular}{lccccc}
\hline & \multicolumn{2}{c}{ Recognition } & & \multicolumn{2}{c}{ Cued Recall } \\
\cline { 2 - 3 } \cline { 5 - 6 } Type of Imagery & First Item & Second Item & & Light Context & Dark Context \\
\hline Interactive & .75 & .82 & & .40 & .31 \\
Successive & .64 & .79 & & .31 & .14 \\
Separate & .73 & .83 & & .05 & .03 \\
$\quad M$ & .71 & .81 & & .25 & .16 \\
Ab Relative Size & .56 & $.80^{*}$ & & .10 & .15 \\
\hline
\end{tabular}

*Invisible item. 
ing. For the other variables, the four instructions differed $[F(3,152)=5.32, M S e=0.011]$, the two items differed $[F(1,152)=101, M S e=0.016]$, and the variables interacted $[F(3,152)=6.71]$; means that differ by .09 are reliably different.

The large items were recognized better when they were in the control conditions (.71) than when they were the visible members that obscured their partners (.56). However, the small items were recognized no more poorly when they were hidden by the first items $(.80)$ than when they were in the control conditions (.81). Easily hidden, small items did not suffer from being hidden, but the large items that were hiding them did. The results argue against the importance of visual properties for recognition.

The next two columns are cued recall, averaged over which item was the cue. There was a slight advantage for first over second items as cues $(.20>.17)[F(1,152)=$ $5.18, M S e=0.010]$, but there were no interactions with the other variables. There were reliable effects of type of imagery $[F(3,152)=35.3]$, context $[F(1,152)=6.26]$, and their interaction $[F(3,152)=4.11, M S e=0.010]$; a difference of .06 between means is reliable.

Recall was worse for imagining invisibly than for the average of the control conditions. Imagining the first item very large so that it obscured its partner led to slightly better recall than imagining separately, as in Experiment 1 .

The instruction to have a dark rather than a light scene had its largest effect with successive images, whose recall required that the cue retrieve the context, which in turn must retrieve the target. There is a double relational jeopardy for contextually mediated recall compared with recall for interactively imagined items, for which the direct interaction between items is the relational basis of recall. Although the interactively imagined pairs suffered from darkness, they remained well above all the other conditions.

\section{Conclusions}

As in Experiment 1, the demand to make the first item large enough to hide the second hurt recognition of the large, visible item, relative to recognition of first items in the control conditions. However, the easily hidden, small second items were recognized equally well in all conditions. A visual account would need to explain why the visible items suffered but the invisible ones did not. Also as in Experiment 1, recall in the invisible condition exceeded that for separate imagery but was well below that for interactive imagery, regardless of which item was the cue.

The manipulation of the imagined lightness or darkness of the scene did not affect recognition of the items. Thus "out of sight, out of mind" is not an appropriate summary. Imagined darkness did, however, result in lower cued recall than did imagined light. Is "out of sight, out of relation" a better summary? We think not. We propose instead that the demand to imagine a dark scene oc- cupies enough of the encoding process that the other memory functions lose fidelity. Item-specific encoding suffers less than relational processing, and successively imagined items suffer most because their recall requires two relations, one from the cue to the context and one from the context to the target.

Dark contexts reduced recall without changing the order of recall within the control conditions. The difference in recall between interactively and separately imagined pairs was far greater than any differences between dark and light contexts.

\section{EXPERIMENT 3}

Subjects in Experiment 3 imagined the members of each pair interactively or separately, in scenes that were brightly lit or obscured by the dark fog of night; dark contexts had no effect on recognition but a slight effect on recall in Experiment 2. The instructions were elaborated, and the subjects were encouraged to follow them even if it was difficult. The aim was to contrast memory for items that are made visible or invisible either separately or interactively. Another manipulation was an instruction to imagine the cues on the test in bright light or in darkness.

The experiment examined memory for items, memory for relations between items, and memory for the contexts in which the items were imagined. Each subject imagined some pairs in light and others in darkness. Each pair was encoded as a single interactive image or as two separate images. On the test, subjects imagined the cues in light or in darkness, discriminated old items from new ones, classified old items as having been studied in light or darkness, and recalled what partners they could.

An extension of our account is useful here. Winograd and Lynn (1979) found that cued recall was not much better for interactive imagery than for separate imagery if each pair had a unique context. For example, monkey and bicycle can be imagined at separate tables in a restaurant or they can be interacting in that context. Begg and Sikich (1984) found that items interacting with each other were recalled well whether or not they were interacting with the context, but separately imagined items were recalled poorly unless each one was interacting with the context, and even then recall fell short of recall for items that were interacting with each other. Begg and Sikich reasoned that interactively imagined items can be recalled when cues retrieve the memory units, but that contextually mediated recall requires two relational steps, one from the cue to the context and the other from the context to the target. Begg and Sikich also found that two items interacting with each other but not with the context were recalled better than two items interacting with each other and the context. They suggested that the more relations a unit represents, the less well it represents them. A useful metaphor from communication theory contrasts bandwidth and fidelity. An encoding has broad bandwidth if it is informative about many specific events, and high fidelity if a par- 
ticular event is precisely encoded. The general case is for a trade-off between bandwidth and fidelity. On average, the more questions an encoded message answers, the less precisely it answers any one of them.

The implication of a trade-off between bandwidth and fidelity is that, on average, the more functions a trace serves, the less well it serves any of them, compared with a trace dedicated specifically to that function. Requiring the trace to be informative about the contextual background reduces the fidelity with which the trace encodes item-specific information and interitem relations. Perhaps imagining objects in the dark is a tacit demand to increase the bandwidth of the trace by including more information than would normally be encoded.

Our account makes many of the same predictions as does a visual account: regardless of whether making the scene dark increases the memory load or makes the tobe-retrieved items invisible, performance should suffer. A difference between the accounts concerns classification of contexts. By a visual account, memory for an item is memory for its context. Therefore, recognized items should be accurately classified, and recall should be better if the classification is correct rather than incorrect. By our account, however, the reduced quality of memory should occur for all measures, and the amount of shortfall on any measure should be unrelated to the amount of shortfall on any other.

In summary, if the visual properties of images affect memory, any measure that includes dark study or dark test should be poor, except the accuracy of classification of recognized items; if recognition is based on retrieved images, the lightness or darkness of the scene should be known when the item is recognized.

We expected that interactive imagery would surpass separate imagery in cued recall in all conditions. Interactive imagery was not expected to exceed separate imagery in recognition or classification, but it might fall short if the demand to imagine items interactively makes encoding context specific.

The expectations for light and dark contexts were more complicated. Experiment 2 found no differences in recognition for items studied in light or in darkness. In Experiment 3, however, each subject had both light and dark scenes to remember; because lightness or darkness discriminates among traces, larger effects of light and dark contexts were expected for recognition in Experiment 3 than in Experiment 2. If the demand to encode dark contexts occupies more of the encoding process than the demand to encode light contexts, then all measures should suffer from darkness, but the suffering should be independent from measure to measure.

\section{Method}

Subjects. Eighty students from the same pool served in eight groups of 10 .

Materials and Procedure. The list had 60 pairs, with the first 6 and last 6 as fillers. The 120 nouns for the pairs and 48 distrac- tors for the test were selected from Paivio et al.'s (1968) norms, with $I>6$ and $\mathrm{F}>10$; all assignments of items to conditions were random.

The list was recorded as in the other experiments, but with a break after the 30th pair. Half the subjects studied the whole list by interactive imagery, and half used separate imagery. All subjects studied half of the items by imagining scenes in which all items were clearly visible in bright sunlight, and half by imagining scenes in which the items were present but could not be seen because of the dark fog of night. The first half of the list was light and the second dark for half the subjects, and the order was reversed for the other subjects. The subjects received the appropriate instructions and studied the first 30 pairs, after which they were reminded to continue with interactive or separate study, but were switched either from light to dark scenes or vice versa.

The test immediately followed study. The subjects were told to imagine all the cues in the light or in the dark, with half receiving each instruction. Of the 96 items, 48 were old; of these, 24 were from each half of the list; of these, 12 were first and 12 were second items. Next to each item were L, D, and $N$. The subjects were to circle $\mathrm{N}$ for new items and to classify the others as having been studied in the light (L) or the dark (D), and were to recall as many partners of the old items as they could. The test was self-paced, and took less than $15 \mathrm{~min}$.

\section{Results and Discussion}

We present the results in sections. All $F$ values have $d f=1,72$.

Recognition and recall. This section ignores the classification of items as having been studied in the light or in the dark; all $M S$ es are less than 0.01. First, imagining the tested cues in the dark hurt memory; light cues did not have higher hit rates than dark cues ( .82 vs. .79), but the false alarm rate was lower ( .17 vs. $.29 ; F=8.09)$, and recall was better ( .19 vs. $.12 ; F=5.46)$. Second, imagining items in the dark during study also hurt memory. For the light-dark order, recognition favored the early light over the late dark (.83 vs. .77). For the dark-light order, recognition favored the late light over the early dark ( .84 vs. $.79 ; F=12.3)$. The interaction was also reliable in recall $(F=8.27)$ : for the dark-light order, recall favored the late light over the early dark (.21 vs. .15), but for the light-dark order, the early light was not much better than the late dark (.15 vs. .12).

There were no interactions between dark and light study and test. The advantage in recognition for the items studied in the light was similar with light cues (.85 vs. .79) and dark cues ( $.83 \mathrm{vs} .76)$. The advantage in recall was also similar with light cues (.22 vs. .13) and dark cues (.16 vs. .10).

Finally, interactive and separate imagery differed in recall $(.25>.05, F=38.3)$, but not in hits ( $.80 \mathrm{vs.} .82)$ or false alarms (.20 vs. .26); no interactions involved the two instructions. Interactive imagery again exceeded separate imagery in recall, even if the items were related in the dark.

In sum, there are costs to recognition and recall of dark study and dark test, and the costs are independent of each other. Images in the light have more item-specific and 
relational information than images in the dark. Interactive imagery surpassed separate imagery in recall in every combination of the other factors.

Classification. This section examines memory for light and dark contexts. Late items were classified more accurately than early ones, (.70 vs. . $59, F=17.6, M S e$ $=0.027$ ). The good accuracy for late items ranged from .70 to .75 , except for interactively studied pairs tested with dark cues (.62); the poor accuracy for early items ranged from .54 to .58 , except for separately studied items tested with light cues (.69); these three variables interacted $(F=6.44)$. On average, recognition of items that were separately imagined was slightly better than that of items that were interactively imagined (.67 vs. $.62, F=$ $3.43, p=.07, M S \mathrm{e}=0.0078$ ), and light cues were better than dark cues $(.68$ vs. $.61, F=5.88)$. Thus, recognition of separately studied items instates better contextual information than does recognition of interactive items, and light cues retrieve traces that are relatively informative about their original contexts. The combination of interactive imagery and dark cues makes recognition of even the late items poor, and the combination of separate imagery and light cues makes recognition of even the early items good.

Were light and dark contexts equally memorable? The analysis is complicated by bias; people called more new items dark than light (.14 vs. $.10, F=7.73, M S e=$ $0.0084)$. This bias was no greater with dark cues $(.17 \mathrm{vs}$. $.13)$ than with light cues (.10 vs. .06), but it did interact with order $(F=9.63)$; new items were equally often called dark as light (.10 vs. .11) in the light-dark order, but more new items were called dark than light (.17 vs. .09 ) in the dark-light order. That is, new items were most often called dark if the early items were darkly encoded. If "dark" was the default classification when no explicit context was retrieved, the accuracy of classification of early dark items was inflated.

Light contexts were remembered better than dark ones, although study order did not interact with early or late items $(F=1.68)$. Recent items studied in light and dark contexts were equal (.71 vs. .70), even considering bias; after the dark-light order, .09 of the new items were called light, and after the light-dark order, .10 of the new items were called dark. Recent items gave no evidence of better memory for light than dark contexts, but early light contexts were remembered better than early dark ones. For the light-dark order, .62 of the early items were correctly called light and .11 of the new items were called light, for a difference of .51. For the dark-light order, .57 of the early items were correctly called dark, but .17 of the new items were called dark, for a difference of .40 . A critical difference of .07 is needed for means to differ reliably.

Thus dark contexts are not remembered as well as light ones, even though the items in this analysis are ones that have been recognized. Recognizing an item does not automatically provide accurate information about its context.
Rather, items studied in the dark are less well associated with their contexts than are items studied in the light.

Conditional Recall. The final concern was with whether there was an association between how the cues were classified and whether recall succeeded. Recall favored interactive over separate study (.29 vs. $.06, F=$ $43.0, M S e=0.0063$ ), and no interactions involved this variable. Recall was better for light than dark cues $(F=$ $6.82)$ and for cues called light rather than dark $(F=10.2$, $M S \mathrm{e}=0.0058)$; these two variables interacted $(F=$ 7.85 ), because the advantage in recall for light cues over dark ones was greater for items called light (.27 vs. .13) than for those called dark (.17 vs. .13). Thus there is some association between recall and whether people think earlier contexts were light or dark, but the results do not indicate whether light traces allow good recall, or whether subjects attribute lightness to good traces.

Is accuracy of classification associated with recall? Accuracy can be seen in the three-way interaction of study order, early and late items, and classification $(F=5.88$, $M S e=0.024)$. For light-studied pairs, recall favored cues called light rather than those called dark, with a larger difference for late pairs (.27 vs. .15) than for early ones (.19 vs. .12). For dark-studied pairs, late ones had better recall if cues were called dark rather than light (.19 vs. .12 ), but early ones were recalled better if the cues were wrongly called light rather than rightly called dark $(.23$ vs. .14).

On average, recall favors pairs whose cues are classified correctly. The association between classification and recall is stronger for light than dark contexts, and for late than early items. However, the few early dark items that were called light were the best recalled of any pairs studied in the dark. It is likely that the subjects called these items light because they could recall the partners; memory for their contexts cannot be the reason for the good recall. Therefore, the association between items is at least partially independent of the association between items and their contexts.

\section{Conclusions}

Experiment 3 is most easily summarized by three contrasts. Interactive and separate imagery differed in cued recall in every combination of manipulations and measures. They did not differ in recognition, but classifications of the original contexts favored the separately imagined items. Thus the two procedures differ in relational processing, and the demand to relate the items may result in a loss of fidelity of other information.

A second contrast is between cues imagined in light or darkness. Dark cues resulted in worse recognition of items, recall of pairs, and memory for contexts. We propose that the demand to encode the cues in the dark increases the bandwidth of the encodings, with a loss in the fidelity of other information.

The third contrast is between pairs originally imagined in light or in dark scenes. Pairs encoded in the dark gener- 
ally suffered relative to those encoded in the light. The cost of dark study occurred for recognition of the items, recall of the pairs, and memory for the early contexts. We propose that the demand to maintain a dark context increases the memory load. A memory record carries in formation about the identity of the cue, the context in which it was encoded, and the partner with which it appeared; all of this information is of better quality after light than dark study.

In summary, the results indicate that a memory record serves many functions, each partially independent of the others. Recognizing an item depends on item-specific information; it does not automatically provide information about its context. The information relating an item to its context is also independent of the interitem relations that enable recall of partners. Tasks that increase the bandwidth of encoding may have costs for the fidelity of some information, but a cost for one set does not cause a cost for any others.

\section{GENERAL DISCUSSION}

These experiments examined the effects of imagining items in ways that make them visually indistinct. One way was to have only one visible item in the image. Although recognition is the measure that, by a visual account, suffers from invisibility, there were no unique costs of invisibility. Instead, both items suffered if either one was invisible. First members of pairs were recognized equally well if they became invisible or stayed visible. Second members were recognized nearly as well as the first ones if they were visible, large, or separate, but suffered if they were hidden by or interactive with the first items.

Recall did not depend on whether the visible or the invisible item was the cue and, unlike recognition, was not worse than average recall in the control conditions. Recall was sensitive to the type of encoding, varying with how much relational processing was needed. Inserting one image into another demands jointly considering the items, and recall after insertion was relatively high. In addition, imagining a second item as enclosing or being in front of an existing image requires less joint consideration than does enclosing the second in the first or imagining the second as being behind, and order affected recall in these conditions.

The other way of making objects visually indistinct was to have subjects imagine them in dark scenes. Items imagined in the dark do not suffer in recognition unless other items in the list are imagined in the light. Imagining items in the dark does, however, hurt cued recall and hurt classification of the lambent character of the imagined scenes.

The results do not imply that visual properties of images are the determinants of item discriminability, interitem associations, and associations between items and their backgrounds. The results indicate that imagining requires the use of permanent knowledge as a source of discriminative information about the items, and the use of perma- nent skills to encode subsets of knowledge pertinent to each item in the context of the other.

Our account explains performance in terms of two causal factors and two demand characteristics. One causal factor is relational processing, which causes the memory units to retain information that links items to each other and to their contexts. If the items are relationally linked, then retrieval of one makes the other one available. The other causal factor is item-specific processing, which causes the memory units to retain information that specifically identifies the items that were processed. Given adequate item-specific information, a cue that retrieves a unit will be identified as an old item.

One demand characterisitic is the extent to which encoding an item in a context is specific to that context. Relational processing may bias encoding so that an item is interpreted to fit the context, and is interpreted differently from the way the item would be encoded if it appears alone as a cue. Such memory failures are caused by the mismatch between the remembered encoding and the encoded cue, not by the fact that items are processed in relation to each other. The demand to process items in relation to each other defines an occasion that has an increased probability of context-specific encoding, but relational processing can be accomplished without context-specific encoding

The other demand characteristic is the tacit demand to increase the bandwidth of memory units. Requiring a unit to retain more information does not cause the unit to be less precise about any piece of information, but it does increase the probability that any particular piece of information will be represented less faithfully than if the unit were dedicated specifically to that piece of information. If a unit retains information about one, two, or three items, we expect recognition to be progressively worse, on average, as the unit has an increase in bandwidth. If a unit retains information about one, two, or three relations, we also expect a decline in recall. The demand to encode items in the dark is a demand to do more than usual, because encoding in the light is the default procedure. Therefore, encoding in the dark, on average, decreases the fidelity of information specific to the items, information that relates the items to each other, and information that relates each item to its context.

An advocate of a visual account can point to some of our findings as support for the account, but must elaborate the account to explain all the results. For example, the invisible second items in Experiment 1 were the most poorly recognized; however, the drop in recognition for those items was not greater than the drop for their visible partners, and recognition of invisible first items was almost identical to that of visible ones. Furthermore, small, easy-to-hide items in Experiment 2 were recognized no more poorly when they were invisible than when they were in the control conditions, but recognition of their large partners was worse when they were the visible partners in a depiction than when they were in the control 
conditions. It addition, imagining pairs in dark scenes hurt all measures of memory, as a visual account would expect, but the losses were independent of each other, denying that any single loss caused any of the others. Our account is consistent with the results, and it makes no reference to the visual nature of the images. It remains for others to explain the results in terms of that visual nature.

\section{REFERENCES}

BAKER, L., SANTA, J. L. (1977a). Context, integration, and retrieval. Memory \& Cognition, 5, 308-314.

BAKER, L., \& SANTA, J. L. (1977b). Semantic integration and context. Memory \& Cognition, $5,151-154$.

BEGG, I. (1973). Imagery and integration in the recall of words. Canadian Journal of Psychology, 27, 159-167.

BEGG, I. (1978a). Imagery and organization in memory: Instructional effects. Memory \& Cognition, 6, 174-183.

BEGG, I. (1978b). Similarity and contrast in memory for relations. Memory \& Cognition, 6, 509-517.

BEGG, I. (1979). Trace loss and the recognition failure of unrecalled words. Memory \& Cognition, 7, 113-123.

BEGG, I. (1982). Imagery, organization, and discriminative processes. Canadian Journal of Psychology, 36, 273-290.

BEGG, I. (1983). Imagery instructions and the organization of memory. In J. C. Yuille (Ed.), Imagery, memory and cognition: Essays in honor of Allan Paivio (pp. 91-115). Hillsdale, IL: Erlbaum.

BEGG, I., ANDERSON, M. C. (1976). Imagery and associative memory in children. Journal of Experimental Child Psychology, 21, 480-489.

BegG, I., Maxwell, D., MitTerer, J. O., \& Harris, G. (1986). Estimates of frequency: Attribute or attribution? Journal of Experimental Psychology: Leaming, Memory, \& Cognition, 12, 496-508.

BeGG, I., SIKICH, D. (1984). Imagery and contextual organization. Memory \& Cognition, 12, 52-59.

BEGG, I., \& YouNG, B. J. (1977). An organization analysis of the form class effect. Journal of Experimental Child Psychology, 23, 503-519.

BoWER, G. H. (1970). Imagery as a relational organizer in associative learning. Joumal of Verbal Learning \& Verbal Behavior, 9, 529-533.

Brooks, L. R. (1968). Spatial and verbal components of the act of recall. Canadian Journal of Psychology, 22, 344-368.

DempSTER, R. N., \& RoHWER, W. D. (1974). Component analysis of the elaborative encoding effect in children's learning. Journal of Experimental Psychology, 103, 400-408.

Glass, A. L., Millen, D. R., BecK, L. G., \& Eddy, J. K. (1985). Representation of images in sentence verification. Joumal of Memory \& Language, 24, 442-465.

Intons-PETERSON, M. J. (1983). Imagery paradigms: How vulnerable are they to experimenters' expectancies? Journal of Experimental Psychology: Human Perception \& Performance, 9, 394-412.

JaCOBY, L. L., Craik, F. I. M., BeGG, I. (1979). Effects of decision difficulty on recognition and recall. Journal of Verbal Learning \& Verbal Behavior, 18, 585-600.
KEEnAN, J. M. (1983). Qualifications and clarifications of images in concealed objects: A reply to Kerr and Neisser. Joumal of Experimental Psychology: Leaming, Memory, \& Cognition, 9, 222-230.

KeEnan, J. M., Moore, R. E. (1979). Memory for images of concealed objects: A reexamination of Neisser and Kerr. Journal of Experimental Psychology: Human Learning \& Memory, 5, 374-385.

KERR, N. H. (1983). The role of vision in "visual imagery" experiments: Evidence from the congenitally blind. Journal of Experimental Psychology: General, 112, 265-277.

KerR, N. H., NeISSER, U. (1983). Mental images of concealed objects: New evidence. Joumal of Experimental Psychology: Learning, Memory, \& Cognition, 9, 212-221.

Kosslyn, S. M., * Pomerantz, J. R. (1977). Imagery, propositions and the form of internal representations. Cognitive Psychology, 9 , 52-76.

LURIA, A. R. (1968). The mind of a mnemonist. New York: Basic Books. MCGEE, R. (1980). Imagery and recognition memory: The effects of relational organization. Memory \& Cognition, 8, 394-399.

NejSSER, U., KERR, N. (1973). Spatial and mnemonic properties of visual images. Cognitive Psychology, 5, 138-150.

Paivio, A. (1975). Perceptual comparisons through the mind's eye. Memory \& Cognition, 3, 635-647.

Paivio, A., Yuille, J. C., \&adigan, S. A. (1968). Concreteness, imagery, and meaningfulness values for 925 nouns. Jourmal of Experimental Psychology Monograph Supplement, 76(1, Pt. 2).

SEGAL, S. J., \& FusELLA, V. (1970). Influence of imaged pictures and sounds on detection of visual and auditory signals. Journal of Experimental Psychology, 83, 458-464.

ShEPARD, R. N., METZLER, J. (1971). Mental rotation of threedimensional objects. Science, 171, 701-703.

WINOGRAD, E., \& LYNN, D. S. (1979). Role of contextual imagery in associative recall. Memory \& Cognition, 7, 29-34.

Zimler, J., \& KeENAN, J. M. (1983). Imagery in the congenitally blind: How visual are visual images? Joumal of Experimental Psychology: Learning, Memory, \& Cognition, 9, 269-282.

\section{NOTE}

1. A note is in order about experimenters' expectancies. IntonsPeterson (1983) compared the performances of subjects tested by experimenters who held different hypotheses, and found differences in performance in the direction of the hypotheses. Two experimenters tested the subjects in the present experiments. Experiment 2 was conducted by Maryann Azzarello, who thought invisible images would be unmemorable, and who expressed disappointment at the results. The other experiments were conducted by an experienced research technician, Ann Anas (A.A.), who worked with Allan Paivio for 6 years and has worked with Ian Begg for 6 years. A.A. conducts experiments in a polished and professional manner, but is certainly not naive about imagery; she insists that all experiments give competing hypotheses a fair chance. Possible outcomes and their meanings were discussed at length before any experiments were conducted.

(Manuscript received April 27, 1987; revision accepted for publication November 30,1987 .) 